\title{
Acanthosis Nigricans and Diabetes Risk Factors: Prevalence in Young Persons Seen in Southwestern US Primary Care Practices
}

\author{
Alberta S. Kong, MD, MPH \\ Robert L. Williams, MD, MPH \\ Melissa Smith, BA ${ }^{2}$ \\ Andrew L. Sussman, PbD, MCRP $P^{2}$ \\ Betty Skipper, $\mathrm{PbD}^{2}$ \\ Andrew C. Hsi, MD, MPH ${ }^{1}$ \\ Robert L. Rbyne, MD \\ On bebalf of RIOS Net clinicians \\ 'Department of Pediatrics, University \\ of New Mexico School of Medicine, \\ Albuquerque, NM \\ ${ }^{2}$ Department of Family and Community \\ Medicine, University of New Mexico \\ School of Medicine, Albuquerque, NM
}

$\sqrt{m_{1}}$

MORE ONLINE
www.annfammed.org

\begin{abstract}
PURPOSE Evidence shows acanthosis nigricans is often associated with hyperinsulinemia and may indicate increased risk of type 2 diabetes mellitus. The purpose of this study was to determine the association of acanthosis nigricans with type 2 diabetes risk factors and disease in young persons.
\end{abstract}

METHODS We conducted a cross-sectional study in the Research in Outpatient Settings Network, a practice-based research network in southwestern US communities. Participating clinicians $(\mathrm{N}=96)$ collected data on children and young adults aged 7 to 39 years seen during a 2-week sampling period. The main outcomes were the prevalence of acanthosis nigricans, type 2 diabetes risk factors (ethnicity, family history of type 2 diabetes, hypertension, overweight/obesity), type 2 diabetes, and the relationships among these.

RESULTS Among 1,133 patients sampled, risk factors for type 2 diabetes were common: $69 \%$ had a family history of the disease; $3 \%$ of children (aged 7 to 19 years) and $12 \%$ of adults had hypertension; $43 \%$ of children and $73 \%$ of adults were overweight or obese; and $80 \%$ were members of ethnic minorities. Acanthosis nigricans was found in $17 \%$ of children and $21 \%$ of adults. Among children and adults alike, the more type 2 diabetes risk factors that were present, the higher the prevalence of acanthosis nigricans $(P<.001)$. The prevalence ratio for type 2 diabetes in patients with acanthosis nigricans was 1.97 (95\% confidence interval, 1.18-3.27; $P=.01$ ) after controlling for age, body mass index, and the number of type 2 diabetes risk factors. Clinicians reported that the identification of acanthosis nigricans frequently led to discussions about lifestyle modification for decreasing the risk of type 2 diabetes.

CONCLUSIONS Patients with acanthosis nigricans are likely to have multiple risk factors for type 2 diabetes. Acanthosis nigricans may be an independent risk factor for this disease. Detection of acanthosis nigricans may help clinicians more rapidly identify high-risk individuals for diabetes counseling.

Ann Fam Med 2007:5:202-208. DOI: 10.1370/afm.678.

\section{INTRODUCTION}

AC Annals Journal Club selection, see inside back cover or http://www. annfammed.org/AJC/.

Conflicts of interest: none reported

\section{CORRESPONDING AUTHOR}

Alberta S. Kong, MD, MPH

Department of Pediatrics

MSC 105590

1 University of New Mexico

Albuquerque, NM 87131-0001

akong@unm.edu
$\mathrm{T}$ ype 2 diabetes mellitus has reached epidemic proportions in the United States, affecting 20.8 million people, or $7 \%$ of the population. ${ }^{1}$ In 2002, this disease cost the nation $\$ 132$ billion dollars, or $10 \%$ of the annual health care budget. Risk factors associated with type 2 diabetes are older age, obesity, family history of the disease, hypertension, dyslipidemia, physical inactivity, and belonging to certain racial/ethnic groups. ${ }^{2,3}$ The greater the number of risk factors a person has, the greater the chance of developing the disease. Lifestyle interventions can prevent or delay disease onset by as much as $58 \%$, making identification of highrisk patients a priority in primary care. ${ }^{4}$

In primary care practices, where competing demands exist within the 
brief patient encounter, a method for rapidly identifying persons at high risk for type 2 diabetes would be useful. Considering the number of recommended preventive care interventions and the time required to deliver these, any shortening of the risk assessment process can be important. ${ }^{5,6}$ Although patients often have well-known type 2 diabetes risk factors (such as obesity or family history), evaluation and treatment for the disease fall short of recommended practice. ${ }^{7}$

Acanthosis nigricans is a dermatologic condition associated in some cases with hyperinsulinemia. ${ }^{8-14}$ Children with this condition are 1.6 times $^{12}$ to 4.2 times $^{11}$ as likely as those without it to have hyperinsulinemia. Acanthosis nigricans is characterized by thickening and darkening of the upper layers of the skin, resulting in a velvety appearance (see Supplemental Figure, available online-only at http://www.

annfammed.org/cgi/content/full/5/3/202/DC1).

Typical areas of involvement include the posterior neck, the axilla, the elbows, and the knees; the neck is involved $93 \%$ to $99 \%$ of the time. ${ }^{15,16}$

The association of acanthosis nigricans with hyperinsulinemia has led to speculation of a possible further association with type 2 diabetes. The natural history of acanthosis nigricans with respect to type 2 diabetes has not been determined, but evidence suggests the former may be a risk factor for the latter. ${ }^{14,16,17}$ A readily apparent, rapidly identifiable physical examination marker identifying patients at increased risk for type 2 diabetes could stimulate discussions of lifestyle modifications in the primary care setting.

We conducted this study to clarify further the relationship of acanthosis nigricans to type 2 diabetes mellitus and its risk factors in patients aged 7 to 39 years. These associations are important because the use of this dermatologic condition in identifying patients at increased risk for diabetes can potentially help direct clinical prevention strategies. The research questions addressed in this study were (1) What is the relationship of acanthosis nigricans to type 2 diabetes risk factors? and (2) Is acanthosis nigricans an independent risk factor for type 2 diabetes?

\section{METHODS}

\section{Study Design and Setting}

We used a cross-sectional study design to describe the prevalence of acanthosis nigricans and other risk factors for type 2 diabetes mellitus in persons aged 7 to 39 years seen in primary care practices in New Mexico. The study was conducted in a practice-based research network, the Research in Outpatient Settings Network (RIOS Net). At the time of the study, RIOS Net comprised 176 clinicians (57\% family physicians,
$23 \%$ pediatricians, 9\% internists, 5.5\% nurse-practitioners, and $5.5 \%$ physician's assistants) practicing in 30 community health centers, 10 Indian Health Service clinics, 11 academic sites, and 5 private practices. Network members serve predominantly low-income, culturally diverse communities. RIOS Net clinicians were recruited into the study through listserv notices, direct contact, and network meetings. Recruitment was designed to produce a sample of clinicians who were geographically and organizationally representative of the overall network membership.

\section{Sample}

Practice staff provided patients a study information sheet while the patients were waiting to be seen. In the examination room, participating clinicians asked all patients aged 7 to 39 years if they would participate. Consecutive 2-week data collection periods were distributed throughout the overall sampling period, from October 2002 to June 2004, to accommodate for seasonal variations in patient visits.

Patients were enrolled only once during the sampling period. Other than age, there were no exclusion criteria. Pregnant women were later excluded from analysis because their weight did not accurately reflect their baseline body mass index (BMI). The protocol was approved by 4 human subjects review boards with jurisdiction over participating RIOS Net practices. We calculated a sample size sufficient to produce an acanthosis nigricans prevalence estimate of 0.5 (which produces a maximum sample size) with $95 \%$ confidence interval widths of 0.05 . The required sample size was 371 for children aged 7 to 19 years and for adults aged 20 to 39 years.

\section{Data Collection}

Data were collected by primary care clinicians at the time of encounter, using either a paper data collection instrument or a personal digital assistant (PDA). Patient demographics; family history of type 2 diabetes in first- or second-degree relatives; personal history of type 2 diabetes, hypertension, or hyperlipidemia by clinician diagnosis; measured height and weight on that $\mathrm{day}_{;}$and presence or absence of acanthosis nigricans on the neck were recorded. Data were collected by the clinician from information available in the chart and by interview of the patient at the time of the visit. Overweight was defined as having a BMI in the 85 th to 94 th percentile range for children and having a BMI of 25 to $29 \mathrm{~kg} / \mathrm{m}^{2}$ for adults; obesity was defined as having a BMI in the 95th percentile or greater for children and having a BMI of $30 \mathrm{~kg} / \mathrm{m}^{2}$ or greater for adults.

Participating clinicians completed Web-based training on acanthosis nigricans before data collection. A 
similar training course was validated on a sample of 13 clinicians using an examination that consisted of 20 photographs of patients who had this condition or other dermatoses. The overall sensitivity and specificity in diagnosing acanthosis nigricans after training were $96 \%(75 / 78)$ and $94 \%(171 / 182)$, respectively.

Before data collection, participating clinicians were personally contacted by RIOS Net staff who provided them with study instruments and protocols; instructed them on the research protocol and use of PDAs for data collection; and helped set up computer systems needed to download data from PDAs. In addition, PDAs were programmed to reject out-of-range responses.

The study protocol was piloted in 3 primary care practices. To validate recorded data, the charts of 10 participating clinicians were subsequently reviewed to determine whether diagnoses conformed to published diagnostic criteria. Of 152 charts sampled, 98.7\% of diagnoses of type 2 diabetes mellitus conformed to American Diabetes Association diagnostic criteria, ${ }^{18}$ 97.4\% of hypertension diagnoses conformed to the Joint National Committee diagnostic criteria, ${ }^{19}$ and $94.7 \%$ of diagnoses of hyperlipidemia conformed to the National Cholesterol Education Program diagnostic criteria. ${ }^{20}$

\section{Statistical Analysis}

Frequencies, confidence intervals, and summary statistics were calculated for all variables. We evaluated bivariate relationships of acanthosis nigricans to variables related to type 2 diabetes (age; sex; ethnicity; family history of type 2 diabetes; personal history of type 2 diabetes, hypertension, or hyperlipidemia; and $\mathrm{BMI})$. Differences in prevalence of multiple diabetes risks between those with and without acanthosis nigricans were estimated using the $\chi^{2}$ test for proportions or the Fisher exact test when appropriate. Log-binomial regression modeling ${ }^{21}$ was conducted to determine prevalence ratios for the outcome, type 2 diabetes mellitus. Independent variables were included in the model when $P<.20$ in the bivariate analysis and were then retained in the model if they were significant $(P<.05)$ in the multivariate log-binomial model. The final model contained acanthosis nigricans, age, BMI, and the number of type 2 diabetes risk factors (hypertension, ethnicity, and family history of the disease).

\section{RESULTS}

\section{Sample}

Ninety-six clinicians representing $55 \%$ of RIOS Net members at the study time participated in the study. Five clinicians declined to participate, and 3 more were unable to complete data collection. Sixty-two percent of clinicians were family practitioners, $23 \%$ were pedia- tricians, $6 \%$ were advanced practice nurses, $4 \%$ were internists, and $4 \%$ were physician's assistants. Fortynine percent of the clinicians were located in Indian Health Service practices; $30 \%$ in University of New Mexico primary care practices; $19 \%$ in community health centers; and $2 \%$ in private practices.

Of 1,533 patients aged 7 to 39 years seen during the 2-week sampling periods, 100 declined to participate; 138 were not approached because of acuity of illness, lack of time, or other reasons; 4 had no explanation for nonparticipation; 90 were pregnant females; and 68 had missing data. Characteristics of the 1,133 patients enrolled (74\% of eligible subjects) are shown in Table 1. Forty-three percent were children between the ages of 7 and 19 years, and 57\% were adults between the ages of 20 and 39 years; the majority of participants were of American Indian (36\%) or Hispanic (31\%) descent, reflecting the ethnicity of RIOS Net practices.

Patient demographics associated with diabetes risk factors in bivariate analyses are displayed in Table 2. For those who knew their health history, 69\% had a family history of type 2 diabetes. American Indians had the highest prevalence of family history $(78 \%)$, followed by Hispanics (69\%). Some $73 \%$ of adults aged 20 to 39 years and $43 \%$ of children aged 7 to 19 years were overweight or obese. In all racial/ethnic groups except the non-Hispanic white group, the prevalence of overweight or obesity exceeded $50 \%$. Adults and males had a higher prevalence of hypertension and hyperlipidemia; however, more than half of the population had an unknown lipid status. Acanthosis nigricans was seen in $17 \%$ of children and $21 \%$ of adults. Its prevalence by racial/ethnic group ranged from $3 \%$ in non-Hispanic whites to $19 \%$ in Hispanics and 28\% in American Indians.

\begin{tabular}{ll}
\hline $\begin{array}{l}\text { Table 1. Characteristics of the Study Sample } \\
(\mathbf{N}=1,133)\end{array}$ & $\%$ (No.) \\
\hline Characteristic & $15.8(179)$ \\
\hline Age, years & $26.8(304)$ \\
$7-11$ & $26.4(299)$ \\
$12-19$ & $31.0(351)$ \\
$20-29$ & \\
$30-39$ & $61.6(698)$ \\
Sex & $38.4(435)$ \\
Female & \\
Male & $35.7(405)$ \\
Race/ethnicity & $30.6(347)$ \\
American Indian & $16.9(192)$ \\
Hispanic & $13.7(155)$ \\
White, non-Hispanic & $3.0(34)$ \\
Other* & \\
Missing data & \\
\hline * Other race/ethnicity included Asian, black, Pacific Islander, or mixed. Five of \\
the 155 patients were not Hispanic or Latino and reported "other race." \\
\hline
\end{tabular}




\begin{tabular}{|c|c|c|c|c|c|}
\hline \multirow[b]{2}{*}{ Variable } & \multicolumn{5}{|c|}{ Prevalence* } \\
\hline & $\begin{array}{c}\text { Family History of Type } 2 \\
\text { Diabetes Mellitus }\end{array}$ & $\begin{array}{l}\text { Overweight or } \\
\text { Obese }\end{array}$ & Hypertension & Hyperlipidemia & $\begin{array}{l}\text { Acanthosis } \\
\text { Nigricans }\end{array}$ \\
\hline Total & $69.2(761 / 1,100)$ & $60.5(685 / 1,133)$ & $8.9(97 / 1,087)$ & $16.6(85 / 513)$ & $19.2(218 / 1,133)$ \\
\hline \multicolumn{6}{|l|}{ Age, years } \\
\hline $7-11$ & $67.4(118 / 175)$ & $41.3(74 / 179)^{\dagger}$ & $1.8(3 / 165)^{\dagger}$ & $7.7(5 / 65)^{\dagger}$ & $19.0(34 / 179)^{\ddagger}$ \\
\hline $12-19$ & $67.2(195 / 290)$ & $44.4(135 / 304)$ & $4.5(13 / 291)$ & $7.5(8 / 106)$ & $15.8(48 / 304)$ \\
\hline $20-29$ & $71.8(211 / 294)$ & $71.9(215 / 299)$ & $7.2(21 / 293)$ & $11.6(15 / 129)$ & $17.4(52 / 299)$ \\
\hline $30-39$ & $69.5(237 / 341)$ & $74.4(261 / 351)$ & $17.8(60 / 338)$ & $26.8(57 / 213)$ & $23.9(84 / 351)$ \\
\hline \multicolumn{6}{|l|}{ Sex } \\
\hline Female & $71.8(491 / 684)^{\ddagger}$ & $59.9(418 / 698)$ & $6.1(41 / 671)^{\dagger}$ & $12.9(41 / 318)^{\S}$ & $19.3(135 / 698)$ \\
\hline Male & $64.9(270 / 416)$ & $61.4(267 / 435)$ & $13.5(56 / 416)$ & $22.6(44 / 195)$ & $19.1(83 / 435)$ \\
\hline \multicolumn{6}{|l|}{ Race/ethnicity } \\
\hline American Indian & $77.6(305 / 393)^{\dagger}$ & $71.4(289 / 405)^{\dagger}$ & $10.4(40 / 386)$ & $16.6(32 / 193)$ & $28.1(114 / 405)^{\dagger}$ \\
\hline Hispanic & $68.8(234 / 340)$ & $59.1(205 / 347)$ & $7.4(25 / 340)$ & $20.6(27 / 131)$ & $19.0(66 / 347)$ \\
\hline White, non-Hispanic & $54.9(100 / 182)$ & $43.2(83 / 192)$ & $7.2(13 / 181)$ & $12.2(10 / 82)$ & $3.1(6 / 192)$ \\
\hline Other & $60.9(92 / 151)$ & $56.8(88 / 155)$ & $9.6(14 / 146)$ & $13.8(12 / 87)$ & $16.1(25 / 155)$ \\
\hline \multicolumn{6}{|c|}{ Note: total numbers of patients for each variable are given in Table 1.} \\
\hline \multicolumn{6}{|c|}{$\begin{array}{l}\text { * Cell entries show percentages with risk factor present followed by (number positive/number for whom information was available). } \\
\dagger P<.001 \text { for } x^{2} \text { comparing all categories for indicated variable. } \\
\text { † } P<.05 \text { for } x^{2} \text { comparing all categories for indicated variable. } \\
\S P<.01 \text { for } \chi^{2} \text { comparing all categories for indicated variable. }\end{array}$} \\
\hline
\end{tabular}

\section{Acanthosis Nigricans and Diabetes Risk Factors}

Children and adults with a family history of type 2 diabetes had more than twice the prevalence of acanthosis nigricans compared with those without such family history (Table 3). The prevalence of acanthosis nigricans increased as BMI increased and was higher in those with hypertension. Only 13 (3\%) of normalweight patients had acanthosis nigricans. Hyperlipidemia was not included in the analysis presented in Table 3 because of a low screening rate among the sampled population.

Among children and adults with more than 1 type 2 diabetes risk factor (family history of type 2 diabetes, overweight, hypertension, and minority race/ethnicity, defined as race/ethnicity other than white, non-Hispanic), the prevalence rate of acanthosis nigricans increased successively with each additional risk factor (Figure 1). The positive likelihood ratio of having acanthosis nigricans in patients with more than 2 risk factors for type 2 diabetes was 8.3 between ages 7 and 19 years and 4.2 between ages 20 and 39 years. Two patients with none of the examined diabetes risk factors had acanthosis nigricans.

\section{Table 3. Prevalence of Acanthosis Nigricans by Type 2} Diabetes Mellitus Risk Factor

\begin{tabular}{|c|c|c|c|c|}
\hline \multirow{2}{*}{$\begin{array}{l}\text { Type } 2 \text { Diabetes } \\
\text { Risk Factor }\end{array}$} & \multicolumn{2}{|c|}{ Aged 7-19 Years } & \multicolumn{2}{|c|}{ Aged 20-39 Years } \\
\hline & No. & $\%(95 \% \mathrm{Cl})$ & No. & $\%(95 \% \mathrm{Cl})$ \\
\hline \multicolumn{5}{|l|}{$\begin{array}{l}\text { Family history of } \\
\text { type } 2 \text { diabetes }\end{array}$} \\
\hline Yes & 313 & $21.4(17.0-26.4)^{*}$ & 448 & $25.2(21.3-29.5)^{*}$ \\
\hline No/unknown & 170 & $8.8(5.0-14.1)$ & 202 & $11.4(7.4-16.6)$ \\
\hline \multicolumn{5}{|l|}{ BMI category ${ }^{\dagger}$} \\
\hline Normal & 274 & $2.6(1.0-5.2)^{*}$ & 174 & $3.4(1.3-7.4)^{*}$ \\
\hline Overweight, not obese & 80 & $11.2(5.3-20.3)$ & 187 & $12.3(8.0-17.9)$ \\
\hline Obese & 129 & $51.2(42.2-60.1)$ & 289 & $37.0(31.4-42.9)$ \\
\hline \multicolumn{5}{|l|}{ Hypertension } \\
\hline Yes & 16 & $68.8(41.3-89.0)^{*}$ & 81 & $37.0(26.6-48.5)^{*}$ \\
\hline No/unknown & 467 & $15.2(12.1-18.8)$ & 569 & $18.6(15.5-22.1)$ \\
\hline \multicolumn{5}{|c|}{$\mathrm{BMI}=$ body mass index; $\mathrm{Cl}=$ confidence interval. } \\
\hline \multicolumn{5}{|c|}{$\begin{array}{l}{ }^{*} P<.001 \text { for comparison of prevalence of acanthosis nigricans between those with and without } \\
\text { the risk factor. } \\
+ \text { For children, normal is defined as less than } 85 \text { th percentile; overweight but not obese is defined } \\
\text { as } 85 \text { th to less than } 95 \text { th percentile; and obese is defined as } 95 \text { th percentile or higher. For adults, } \\
\text { normal is defined as } 25 \mathrm{~kg} / \mathrm{m}^{2} \text {; overweight but not obese is defined as } 25 \text { to } 30 \mathrm{~kg} / \mathrm{m}^{2} \text {; and obese } \\
\text { is defined as } \geq 30 \mathrm{~kg} / \mathrm{m}^{2} \text {. }\end{array}$} \\
\hline
\end{tabular}

\section{Type 2 Diabetes}

The overall prevalence of type 2 diabetes in this sample was $6.2 \%$; the prevalence increased as age increased. Ethnic minorities had a higher prevalence than nonminorities $(7.3 \%$ vs $1.0 \%, P<.001)$, and American Indians had the highest prevalence (9.6\%). Patients with a family history of type 2 diabetes had almost 


\section{Figure 1. Prevalence of acanthosis nigricans by number of diabetes risk factors.}

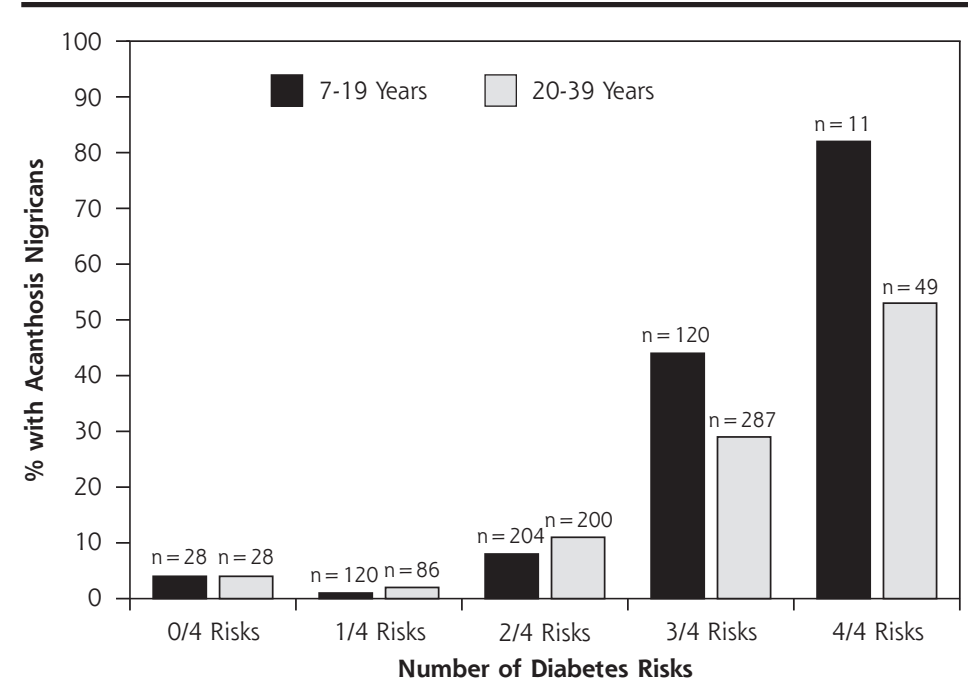

Note: Risk factors included family history of type 2 diabetes mellitus, overweight/obesity, hypertension, and minority ethnicity.

4 times the prevalence of the disease, and those with hypertension had close to 10 times the prevalence, compared with those without the respective risk factor. The prevalence was $12 \%$ in obese individuals, $3.4 \%$ in overweight individuals, and $2.5 \%$ in normal-weight individuals. Type 2 diabetes was present in $15 \%$ of patients with acanthosis nigricans but only $4 \%$ of those without it $(P<.001)$.

\section{Acanthosis Nigricans and Type 2 Diabetes}

Patients with diagnosed type 2 diabetes had a higher prevalence of acanthosis nigricans compared with patients without that diagnosis $(47 \%$ vs $17 \%, P<.001)$. Only $5(1 \%)$ of the children had a diagnosis of type 2 diabetes; 3 of the 5 had acanthosis nigricans $(P=.04)$. Of 650 adults, 65 (10\%) had type 2 diabetes; $46 \%$ of those had acanthosis nigricans, compared with $18 \%$ of their nondiabetic counterparts $(P<.001)$.

Acanthosis nigricans was independently associated with type 2 diabetes after controlling for age, BMI, and the number of other examined diabetes risk factors (Table 4). Patients with acanthosis nigricans were 1.97 times as likely as those without it to have type 2 diabetes $(P=.01)$. Patients who were older, obese, and had more than 1 risk factor were also more likely to have the disease.

\section{An Unanticipated Finding}

We received reports from a number of participating clinicians that the identification of acanthosis nigricans led to discussions about lifestyle modification that would not have otherwise occurred. Awareness of acanthosis nigricans and its possible association with type 2 diabetes reportedly changed their practice behaviors or changed patient receptivity to discussion about risk reduction for diabetes. Because this outcome was unanticipated, our design did not allow us to systematically study this behavioral phenomenon; however, we report it here as a result of potential importance.

\section{DISCUSSION}

We found acanthosis nigricans to be associated with having multiple risk factors for type 2 diabetes mellitus in patients aged 7 to 39 years. As the number of these risk factors increased, so did the prevalence of acanthosis nigricans in both children and adults. The positive likelihood ratio of having this dermatologic condition in patients with more than 2 risk factors for type 2 diabetes was 8.3 for ages 7 to 19 years and 4.2 for ages 20 to 39 years.

Most studies have found acanthosis nigricans to be associated with insulin resistance or hyperinsulinemia, major factors in the pathophysiology of type 2 diabetes, in a substantial proportion of patients. ${ }^{8,9,11-14,22,23}$ Although our study did not measure insulin levels, we found that acanthosis nigricans was independently associated with diagnosed type 2 diabetes. Patients with this dermatologic condition were 1.97 times more

Table 4. Type 2 Diabetes Mellitus Prevalence
Ratios for Factors Related to Type 2 Diabetes
Mellitus, by Log-binomial Regression Analysis

\begin{tabular}{lll}
\hline Factor & $\begin{array}{c}\text { Prevalence } \\
\text { Ratio }(95 \% \mathrm{Cl})\end{array}$ & $\boldsymbol{P}$ Value \\
\hline $\begin{array}{l}\text { Age, years } \\
\text { 7-19 }\end{array}$ & 1.00 & $<.001$ \\
$20-39$ & $7.64(3.17-18.42)$ & \\
Acanthosis nigricans & & \\
No & 1.00 & .01 \\
Yes & $1.97(1.18-3.27)$ & \\
BMl category & & \\
Normal or overweight & 1.00 & \\
Obese & $2.25(1.29-3.91)$ & \\
Number of risk factors* & & \\
0-1 & 1.00 & \\
2-3 & $3.41(1.65-7.06)$ & \\
\hline
\end{tabular}

$\mathrm{Cl}=$ confidence interval; $\mathrm{BMI}=$ body mass index.

* Risk factors included family history of type 2 diabetes mellitus, hypertension, and minority ethnicity. 
likely to have type 2 diabetes compared with their counterparts without it, after controlling for age, BMI, and the number of type 2 diabetes risk factors (hypertension, ethnicity, and family history of the disease). The strength of this association may be understated in these data because we included only patients who had already received the diagnosis of type 2 diabetes.

Clinical detection of acanthosis nigricans may help identify individuals at high risk for type 2 diabetes mellitus ${ }^{24}$ because, as noted, acanthosis nigricans is associated with hyperinsulinemia in a proportion of those with this skin condition. Our study supports its association with type 2 diabetes risk factors and disease.

Although obesity and family history are wellknown risk factors for type 2 diabetes, they do not lead to screening for the disease at rates approaching their prevalence in primary care. Drobac et $\mathrm{al}^{7}$ found that screening laboratory tests were ordered for only $38 \%$ of children who met screening criteria of the American Diabetes Association for type 2 diabetes. They found that a family history of the disease was frequently not recognized. In addition, in that study of 997 children, BMI was calculated for only 92 children and BMI percentiles were plotted for only 10 children. Similarly, Dorsey et $\mathrm{al}^{25}$ found that BMI was recorded by clinicians in only $0.5 \%$ (3/600) of medical records and that rates of undiagnosed and untreated overweight among children were $79.5 \%$ and $83.1 \%$, respectively. Cook et al ${ }^{26}$ examined 32,930 ambulatory visits from the 1997 to 2000 National Ambulatory Medical Care Survey and the National Hospital Ambulatory Medical Care Survey, and found that obesity was diagnosed at only $0.78 \%$ of all visits and $0.93 \%$ of well-child visits. These studies show that well-known risk factors for type 2 diabetes mellitus do not lead to recommended actions to detect the disease.

Although Drobac et $\mathrm{al}^{7}$ found limited screening for type 2 diabetes in the presence of traditional risk factors for the disease, they also found that 93\% of patients with acanthosis nigricans were screened. The report from our clinicians that the detection of acanthosis nigricans stimulated discussions of diabetes prevention suggests that the presence of this condition may somehow change the dynamic of the primary care encounter. Further research is needed to confirm this observation and to determine how the presence of acanthosis nigricans influences clinician decisions to take time to discuss type 2 diabetes risk reduction (eg, by changing patient receptivity to discussions of lifestyle modification).

A recent publication identified factors influencing clinicians' decisions to include obesity prevention counseling in the brief primary care encounter and may help to explain the clinician response to tradi- tional diabetes risk factors. ${ }^{27}$ Clinicians' decisions about time allocation and decisions to engage in preventive counseling were based on multiple stable and situational factors, including the lack of motivation in their patients. In the face of competing demands within the brief encounter, the observable nature of acanthosis nigricans may influence patient motivation.

\section{Limitations}

Some limitations to this study should be noted. First, health history was self-reported. Obtaining family history by self-report is standard clinical practice, however, so our method reflects the usual clinical approach. Second, the study design was cross-sectional and therefore limits our ability to determine the natural history of acanthosis nigricans with respect to type 2 diabetes. Third, because our design did not include blood sampling for diabetes, we were not able to identify those who had undiagnosed disease, thereby possibly underestimating its true prevalence among both those with and those without acanthosis nigricans. Fourth, because our protocol did not require that blood samples be drawn to establish the diagnosis of hyperlipidemia, and more than one half of the study population an had unknown lipid status, the prevalence of known hyperlipidemia may be underestimated because those with an unknown lipid status were categorized as not having hyperlipidemia. Because of the missing data on lipid status, we did not include hyperlipidemia in our analysis of the relationships between type 2 diabetes risk factors and acanthosis nigricans. Finally, because of the nature of southwestern US communities represented in RIOS Net, Hispanic and American Indian persons were overrepresented in our sample. Acanthosis nigricans is less common in nonHispanic whites, and the relationships we observed between acanthosis nigricans and type 2 diabetes risk factors and disease may not be generalizable to this group. Further studies are needed to confirm our findings in other populations.

In conclusion, acanthosis nigricans can be used to rapidly identify those patients with multiple risk factors for type 2 diabetes mellitus. Because lifestyle changes have been proven to reduce the incidence of type 2 diabetes in high-risk adults, acanthosis nigricans may provide primary care clinicians with an efficient method for identifying patients who would most benefit from lifestyle modification interventions. Detection of acanthosis nigricans may also enhance patient and clinician receptivity to discussing risk reduction for type 2 diabetes. Studies are needed to investigate the natural history of acanthosis nigricans, its relationship to the development of type 2 diabetes, and how its presence influences screening and counseling for this disease. 
To read or post commentaries in response to this article, see it online at http://www.annfammed.org/cgi/content/full/5/3/202.

Key words: Acanthosis nigricans; diabetes mellitus; practice-based research network; primary care; screening; risk factors; risk reduction

Submitted February 6, 2006; submitted, revised, November 22, 2006; accepted November 27, 2006.

Findings from this study were presented at the North American Primary Care Research Group Annual Meeting, October 12, 2004, Orlando, Fla.

Funding support: This project was funded through a grant from the American Diabetes Association (1-03-CR-34) and by a grant from the Agency for Healthcare Research and Quality (R21 HS13496).

\section{References}

1. American Diabetes Association. Diabetes statistics. 2006. Available at: http://www.diabetes.org/diabetes-statistics.jsp. Accessed: 28 June 2006

2. American Diabetes Association. Screening for type 2 diabetes. Diabetes Care. 2004;27(Suppl 1):S11-S14

3. Centers for Disease Control and Prevention. National diabetes fact sheet: general information and national estimates on diabetes in the United States. 2003. Available at: http://www.cdc.gov/diabetes/ pubs/factsheet.htm. Accessed: 28 June 2006.

4. American Diabetes Association and National Institute of Diabetes, Digestive and Kidney Diseases. The prevention or delay of type 2 diabetes. Diabetes Care. 2002;25(4):742-749.

5. Crabtree BF, Miller WL, Tallia AF, et al. Delivery of clinical preventive services in family medicine offices. Ann Fam Med. 2005;3(5):430-435.

6. Yarnall KS, Pollak KI, Ostbye T, Krause KM, Michener JL. Primary care: is there enough time for prevention? Am J Public Health. 2003;93(4):635-641.

7. Drobac S, Brickman W, Smith T, Binns HJ. Evaluation of a type 2 diabetes screening protocol in an urban pediatric clinic. Pediatrics. 2004;114(1):141-148.

8. Hud JA Jr, Cohen JB, Wagner JM, Cruz PD Jr. Prevalence and significance of acanthosis nigricans in an adult obese population. Arch Dermatol. 1992;128(7):941-944.

9. Kobaissi HA, Weigensberg MJ, Ball GD, et al. Relation between acanthosis nigricans and insulin sensitivity in overweight Hispanic children at risk for type 2 diabetes. Diabetes Care. 2004;27(6):1412-1416.

10. Litonjua P, Pinero-Pilona A, Aviles-Santa L, Raskin P. Prevalence of acanthosis nigricans in newly-diagnosed type 2 diabetes. Endocr Pract. 2004;10(2):101-106.

11. Mukhtar Q, Cleverley G, Voorhees RE, McGrath JW. Prevalence of acanthosis nigricans and its association with hyperinsulinemia in New Mexico adolescents. J Adolesc Health. 2001;28(5):372-376.
12. Stoddart ML, Blevins KS, Lee ET, Wang W, Blackett PR. Association of acanthosis nigricans with hyperinsulinemia compared with other selected risk factors for type 2 diabetes in Cherokee Indians: the Cherokee Diabetes Study. Diabetes Care. 2002;25(6):1009-1014.

13. Stuart (A, Pate CJ, Peters EJ. Prevalence of acanthosis nigricans in an unselected population. Am J Med. 1989;87(3):269-272.

14. Stuart CA, Smith MM, Gilkison CR, Shaheb S, Stahn RM. Acanthosis nigricans among Native Americans: an indicator of high diabetes risk. Am J Public Health. 1994;84(11):1839-1842.

15. Burke JP, Hale DE, Hazuda HP, Stern MP. A quantitative scale of acanthosis nigricans. Diabetes Care. 1999;22(10):1655-1659.

16. Stuart CA, Gilkison CR, Smith MM, et al. Acanthosis nigricans as a risk factor for non-insulin dependent diabetes mellitus. Clin Pediatr (Phila). 1998;37(2):73-79.

17. Burke JP, Duggirala R, Hale DE, Blangero J, Stern MP. Genetic basis of acanthosis nigricans in Mexican Americans and its association with phenotypes related to type 2 diabetes. Hum Genet. 2000;106(5):467-472.

18. American Diabetes Association. Diagnosis and classification of diabetes mellitus. Diabetes Care. 2006;29(Suppl 1):S43-S48.

19. Chobanian AV, Bakris GL, Black HR, et al. The seventh report of the Joint National Committee on Prevention, Detection, Evaluation, and Treatment of High Blood Pressure: the JNC 7 report. JAMA. 2003;289(19):2560-2572.

20. National Cholesterol Education Program (NCEP) Expert Panel on Detection, Evaluation, and Treatment of High Blood Cholesterol in Adults (Adult Treatment Panel III). Third report of the National Cholesterol Education Program (NCEP) Expert Panel on Detection, Evaluation, and Treatment of High Blood Cholesterol in Adults (Adult Treatment Panel III) final report. Circulation. 2002;106(25):3143-3421.

21. Spiegelman D, Hertzmark E. Easy SAS calculations for risk or prevalence ratios and differences. Am J Epidemiol. 2005;162(3):199-200.

22. Copeland K, Pankratz K, Cathey V, et al. Acanthosis nigricans, insulin resistance (HOMA) and dyslipidemia among Native American children. J Okla State Med Assoc. 2006;99(1):19-24.

23. Yamazaki $\mathrm{H}$, Ito $\mathrm{S}$, Yoshida $\mathrm{H}$. Acanthosis nigricans is a reliable cutaneous marker of insulin resistance in obese Japanese children. Pediatr Int. 2003;45(6):701-705.

24. Hardin DS. Screening for type 2 diabetes in children with acanthosis nigricans. Diabetes Educ. 2006;32(4):547-552.

25. Dorsey KB, Wells C, Krumholz HM, Concato JC. Diagnosis, evaluation, and treatment of childhood obesity in pediatric practice. Arch Pediatr Adolesc Med. 2005;159(7):632-638.

26. Cook S, Weitzman M, Auinger P, Barlow SE. Screening and counseling associated with obesity diagnosis in a national survey of ambulatory pediatric visits. Pediatrics. 2005;116(1):112-116.

27. Sussman AL, Williams RL, Leverence R, Gloyd PW Jr, Crabtree BF. The art and complexity of primary care clinicians' preventive counseling decisions: obesity as a case study. Ann Fam Med. 2006;4(4):327-333. 